\title{
Novel VacA S1d Signal Sequence Found in Helicobacter Pylori from Mexican Children with Recurrent Abdominal Pain
}

\author{
Gabriela Delgado-Sapién ${ }^{1}$, Rene Cerritos ${ }^{2}$, Gerardo González-Valencia ${ }^{3}$, Jose L Mendez ${ }^{1}$, \\ Alejandro Cravioto ${ }^{4}$ and Rosario Morales-Espinosa ${ }^{1 *}$ \\ ${ }^{1}$ Department of Microbiology and Parasitology, Faculty of Medicine, Universidad Nacional Autónoma de México, Mexico City, Mexico. \\ ${ }^{2}$ Research Coordination, Faculty of Medicine. Universidad Nacional Autónoma de México, Mexico City, Mexico. \\ ${ }^{3}$ Hospital of Pediatrics, National Medical Center Siglo XXI. Mexican Social Security Institute. Mexico City, Mexico. \\ ${ }^{4}$ Independent Consultant, Mexico City, Mexico.
}

Received: 31 May, 2016; Accepted: 01 March, 2017; Published: 11 March, 2017

*Corresponding author: Rosario Morales-Espinosa, Department of Microbiology and Parasitology, Faculty of Medicine, Universidad Nacional Autónoma de México, Mexico City, Mexico. Tel: +525 5523 2135; Fax: 5255623 2114; E-mail: marosari@unam.mx

\begin{abstract}
Helicobacter pylori is the causal agent of various gastric diseases and its virulence is associated with high genetic diversity at both a chromosomal and gene level. Genotype I strains are described as being highly virulent. The vacA gene is highly polymorphic and presents different subtypes with each group of alleles apparently having a specific geographic localization. In Mexico, as in other parts of Latin-America, s1b/m1 allele strains are predominant, although other subtypes have also been found. However, there are strains that could not be characterized using their vacA alleles suggesting even more variability. The aim of this study was to determine the Signal Sequence (ss) type of $23 \mathrm{H}$. pylori strains isolated from children with recurrent abdominal pain, which could not be characterized according to the vacA ss using Atherton's criteria. Five strains were sequenced from their ss region. The ss analysis showed the substitution of two base pairs at the 3' end of the complementary sequence for the SS3-F primer alignment site. Based on sequence analysis, we designed a primer set, which was used in a PCR protocol to determine the ss type presented in strains obtained from children. All of the strains yielded a PCR product. Genealogic and population structure analyses of the ss region of our strains with respect to the s1a, s1b and s1c subtype revealed a novel ss subtype, which we named s1d. Although the s1d and s1b subtypes are closely related, both s1b and s1d subtypes have perfectly separated lineages. The s1d subtype is exclusive in LatinAmerican populations. The cytotoxic activity of the s1d/m1 allele was variable, as was seen with the $\mathrm{s} 1 \mathrm{~b} / \mathrm{m} 1$ allele. In conclusion, this present study identified a new vacA signal sequence subtype in H. pylori strains isolated from Mexican children with recurrent abdominal pain. Genealogic analysis showed that s1d subtype of $v a c A$ is a homogenous group and is present only in Latin American populations.
\end{abstract}

Keywords: vacA s1d signal sequence; Helicobacter pylori; Children; Recurrent abdominal pain

\section{Introduction}

Helicobacter pylori is a gram-negative, microaerophilic bacterium that colonizes the human gastric mucosa; it is one of the most common chronic human infections. H. pylori play an important role in the pathogenesis of peptic ulcer disease, distal gastric adenocarcinoma and gastric lymphoma [1]. Disease development results from a number of contributing factors, such as host susceptibility, environmental factors and bacterial genotype [2].

Several virulence factors have been associated with different gastric pathologies, some of these are: urease and catalase production; the presence of adhesins, such as BabA [2,3]; cag pathogenicity island (cag-PAI) [4,5]; CagA expression [6,7]; and the production of a vacuolating cytotoxin encoded by the vacA gene [8]. The variability of its genome is due to its ability to transform naturally and the incorporation of foreign DNA into its genome. The $v a c A$ gene has a mosaic structure consisting of several regions that are conserved and other regions that are highly divergent. Among the divergent regions of the vacA gene, there are basically two regions: the signal sequence (ss) region; and the middle region. For the signal sequence region, four subtypes of vacA have been described (s1a, s1b, s1c, and s2), and for the middle region, two subtypes ( $\mathrm{m} 1$ and $\mathrm{m} 2$ ), all of which can be differentiated using PCR sequencing [8]. All combinations of the vacA signal sequence and middle allele types have been described worldwide. Extensive studies have shown that the vacA sequence signal types have a specific geographic distribution and some genotypes are more associated with disease than others [57]. In particular, the vacA s1c allele is more frequent in people from East Asia $[9,10]$. In Mexico, as in the rest of Latin America, the vacA s1b type is more frequent [11-15], while in most of the Western countries, vacA s1a type is more prevalent [5-7].

In a previous studies performed by our group, the vacA gene of $H$. pylori strains isolated from adults and children were characterized and vacA s1b/m1 allele was found to be the most prevalent in the studied population [11,13]. However, in this same study, we found strains isolated from children that could 
not be characterized according to the subtypes of the vacA gene signal sequence. With this in mind, we considered that there could be a greater variability in this region than had been described previously. In the current study, we randomly chose five strains isolated from children with recurrent abdominal pain that could not be formally typed according to the vacA signal sequence. Based on sequence analysis, we designed a new primer and completed the ss characterization using PCR.

\section{Materials and Methods}

Strains and culture: Using a PCR-based typing system developed by Atherton, we reported the previous characterization of $H$. pylori strain populations isolated from children and adults according to the vacA allele, and other virulence genes, such as $\operatorname{cag} A$ and $\operatorname{vapD}$. In the aforementioned study that was carried out previously by our group, we found that $10 \%$ of the strains (23) could not be typed according to their vacA ss [11,13]. In the current study, we used these $23 \mathrm{H}$. pylori isolates, all of which were isolated from children with Recurrent Abdominal Pain (RAP). Primer sets were used to determine the vacA s1 or s2 signal sequence type according to the Atherton's characterization [8]. Additionally, we used four H. pylori strains as controls for each vacA allele: the 60190 and 26695 strains, which are both $\mathrm{s} 1 \mathrm{a} / \mathrm{m} 1$; the 84183 strain, which is $\mathrm{s} 1 \mathrm{~b} / \mathrm{m} 1$; and the Tx30a strain, which is $\mathrm{s} 2 / \mathrm{m} 2$.

Stock cultures of the $H$. pylori strains stored at $-70^{\circ} \mathrm{C}$ were regrown individually on Casman agar plates supplemented with $10 \%$ sheep blood. The plates were incubated at $37^{\circ} \mathrm{C}$ under microaerobic conditions [GasPakTM EZ Campy (Beckton Dikinson)] for a maximum of 5 days.

DNA Isolation: Each H. pylori strain was cultured as described above. DNA was isolated from the cultured bacteria by harvesting cells from a plate in phosphate-buffered saline solution followed by centrifugation. The pelleted cells were resuspended in $200 \mu \mathrm{l}$ of proteinase K solution (10 mM Tris- $\mathrm{HCl}$ [pH 7.8], 5 mM EDTA, $0.5 \%$ Sodium Dodecyl Sulfate [SDS] and $50 \mathrm{mg}$ of proteinase $\mathrm{K}$ per $\mathrm{ml}$ ), the mixture was then incubated at $55^{\circ} \mathrm{C}$ for $30 \mathrm{~min}$. The DNA was extracted using phenol-chloroform-isoamyl alcohol following standard procedures [16]. DNA was precipitated by adding $1 / 10$ volume of $3 \mathrm{M}$ sodium acetate (pH 5.2) and 2 volumes of ethanol. After centrifugation, the DNA pellet was washed with $70 \%$ ethanol and dried at room temperature. The DNA was dissolved in $50 \mu \mathrm{l}$ of TE buffer (10mM Tris- $\mathrm{HCl}$ [pH8.3], $0.1 \mathrm{mM}$ EDTA) and its concentration was determined by a spectrophotometer (GENios, TECAN Austria GMBH).

vacA typing by allelic type-specific PCR primers: vacA signal sequence typing was performed by PCR with the VA1-F and VA1-R primers to categorize this region as type s1 or s2 [8]. Subsequently, we made individual PCRs to determine the family type of $\mathrm{m} 1$ or $\mathrm{m} 2$ mid-region according to Atherton's protocol using m1-specific primers (VA3-F and VA3-R) and m2-specific primers (VA4-F and VA4-R) [8].

vacA signal sequence sequencing: We randomly selected five strains $(72 \mathrm{a} 10,536 \mathrm{~A} 8,268 \mathrm{c} 3,92 \mathrm{a} 5,64 \mathrm{c} 1)$ from children whose vacA signal sequence could not be typed previously using Atherton's criteria; a 623 bp vacA fragment (coordinates 519 to $1142 \mathrm{bp}$ in the published vacA sequence of strain 60190) corresponding to the 5' end of the vacA gene was amplified by PCR for each strain using CYSS primers (5'-CGCTTTGATGGACACCCCCACAA-3') and new primer (5'-CCCATCCCAACCTCCATCAATCT-3') designed in this study [8]. PCR products were purified using the QIAquick PCR purification kit from QIAGEN and sequenced on both strands. The DNA sequence was analyzed using the LASERGENE (DNASTAR, Inc.) software package. The detailed alignments were performed using the CLUSTALW program. Representative sequences have been deposited in GenBank: Mx536A8 strain [GenBank/EMBL accession no. DQ061145], Mx92a5 strain [GenBank/EMBL accession no. DQ061150], Mx64c1 strain [GenBank/EMBL accession no. DQ061153], Mx72A10 strain [GenBank/EMBL accession no. DQ061154] and Mx268c3 strain [GenBank/EMBL accession no. DQ061156].

Based on sequence analysis, we designed a new SS5-F specific primer (5'-AGCGCCATACCGCAACAAA), which was used together with reverse primer VA1-R, as described previously, to complete the signal sequence genotyping of the strains isolated from children with RAP [8]. All PCR mixtures consisted of $10 \mathrm{ng}$ of $H$. pylori DNA and $5 \mu \mathrm{l}$ PCR buffer 10X solution (Gibco BRL, Gaithersburg, MD), $1.5 \mathrm{mM} \mathrm{MgCl} 2,25 \mathrm{pmol}$ of each primer, 10 nM dNTPs and $5 \mathrm{U}$ of Taq polymerase (Gibco BRL). Conditions for thermal cycling were: 35 cycles at $94^{\circ} \mathrm{C}$ for $1 \mathrm{~min}, 52^{\circ} \mathrm{C}$ for $1 \mathrm{~min}$, and $72^{\circ} \mathrm{C}$ for $1 \mathrm{~min}$. The products were electrophoresed on a $1 \%$ agarose gel and stained with ethidium bromide.

Genealogic and Population Structure Analyses: In order to assess the genealogic relationship between the vacA signal sequence types previously reported in the database GenBank and the ss sequences of our strains, a network of haplotypes was created. Genealogies based on individual allele (haplotypes) sequences were reconstructed using TCS 1.21 software (44). The haplotype network shows the connection between each of the alleles, inferring the number of mutational steps between them (when there is more than one mutational step between one haplotype and another, the existence of an intermediate haplotype can be assumed, which is represented by a small white circle (Figure 1) between the connecting axes). The haplotypes network was constructed using ss sequences from $127 \mathrm{H}$. pylori strains of different subtypes (s1a, s1b, s1c and s2) deposited in GenBank of the National Center of Biotechnology Information (NCBI) and the ss of our 5 sequenced strains. Geographic distribution of vacA allelic types of $H$. pylori was also included in this analysis.

The population structure analysis was made using the linkage model of Bayesian clustering algorithm "STRUCTURE" (45). Analysis was carried out in order to explore how local haplotypes were grouped into subpopulations, and shows the ancestry proportion of each of the subpopulation formed. This technique employed a Bayesian approach to establish the probability of the number of populations in a sample of sequences. This algorithm 


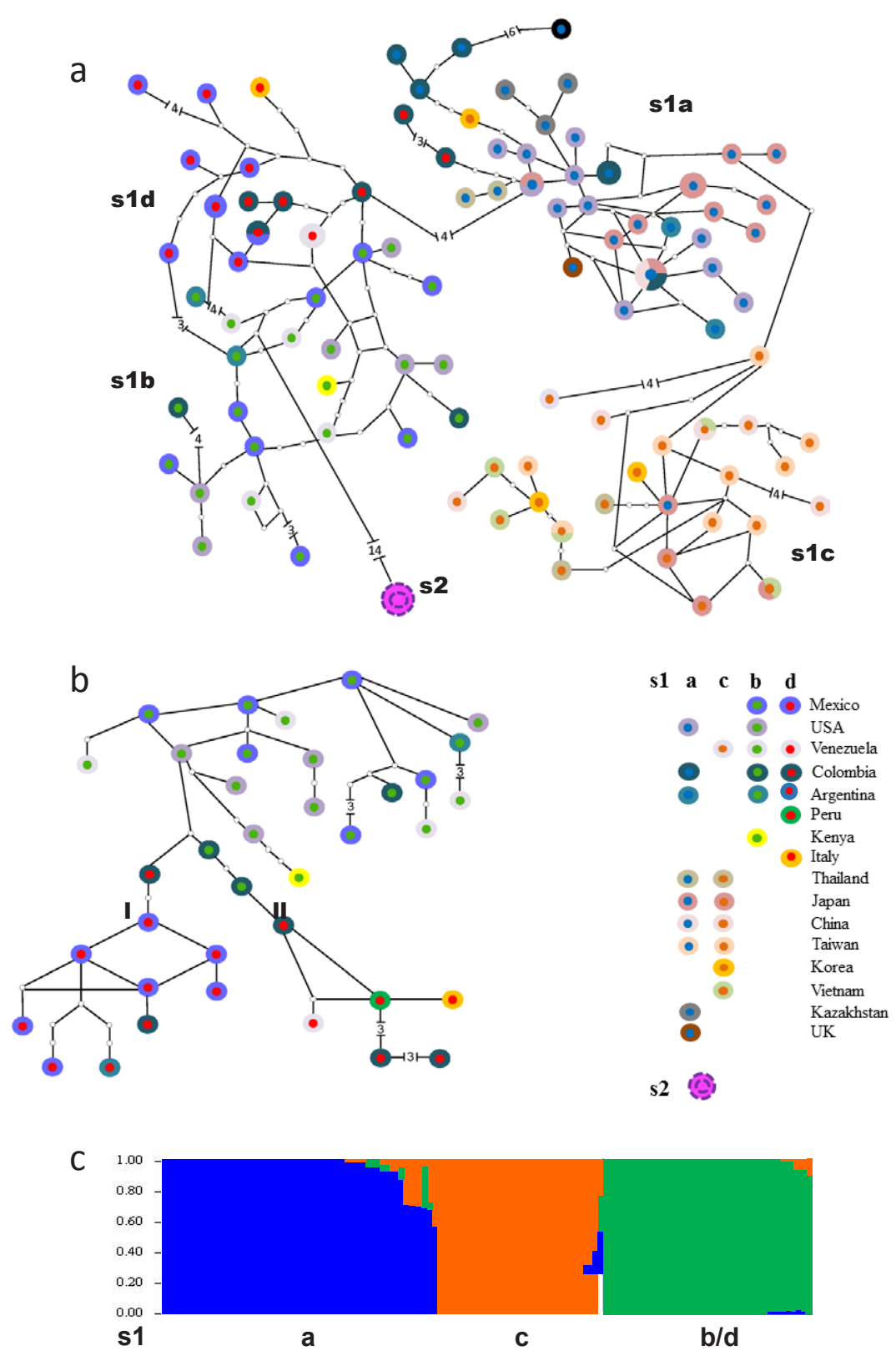

Figure 1: Genealogic relationship of the vacA signal sequence from 132 strains of Helicobacter pylori isolated from different parts of world. a. It shows the network of haplotypes that form 5 well defined populations (s1a, s1b, s1c, s1d and s2). The numbers between bars show the mutational steps that diverge between one subpopulation and other; the small empty circles indicate a hypothetical intermediate haplotype. The subpopulation s2 type diverges about 14 mutational steps from subpopulations s1b / s1d. The subpopulations s1a and s1c (phenotypically cytotoxic) diverge from subpopulations s1b / s1d (less cytotoxic) to 4 mutational steps.

b. It shows two clades, which diverge from s1b to a mutational step. Clade I groups all s1d haplotypes of $H$. pylori strains isolated from children with recurrent abdominal pain in Mexico. Clade II corresponds to strains isolated from other Latin American countries.

c. Results of Structure analysis assuming 3 subpopulations, each color represent one subpopulation: blue for s1a, orange for s1c and green for $\mathrm{s} 1 \mathrm{~b} / \mathrm{d}$.

was run assuming a $\mathrm{K}$ value between 2 and 7 populations (15 replicates per value of $\mathrm{K}$ ) with a burning of 50,000 iterations and 100,000 MCMC subsequent iterations. We found an optimal $\mathrm{K}$ value of 3 , which defined 3 subpopulations (s1a, s1c and s1b/ s1d) very well.
Assessment of vacuolating activity: Each strain was cultured as described earlier. A swab of plate-grown bacteria was then used to inoculate $25 \mathrm{ml}$ of brucella broth with $10 \%$ fetal calf serum. The culture was grown for $48 \mathrm{~h}$ under microaerobic conditions (as described above) with gentle agitation. Culture 
supernatants were concentrated 40 -fold by ultrafiltration as describe previously [17]. The concentrate supernatants were incubated in AGS cells in two-fold serial dilutions. Cells were observed for up to $48 \mathrm{~h}$ to identify the development of vacuoles. The maximum dilution of the sample that produced vacuolation in more than $30 \%$ of the AGS cells was defined as the cytotoxin activity score for a sample. Non-inoculated broth was used as a negative control, and broth culture concentrates from strains 60190 and Tx30a were used as positive and negative vacuolating controls, respectively.

\section{Results}

In a previous study, we reported on the characterization of the vacA genotypes from $H$. pylori strains isolated from patients with different gastric pathologies, where the $\mathrm{s} 1 \mathrm{~b} / \mathrm{m} 1$ vacA allele was the most frequently observed in $H$. pylori strains found in both the antrum and corpus $[11,13]$. However, using Atherton's characterization, the vacA signal sequence was non-typeable in approximately $10 \%$ of the strains, with the majority of typing being achieved in strains isolated from children with recurrent abdominal pain [8].

The characterization of all strains by PCR using the conserved primers VA1-F and VA1-R were typed as $\mathrm{s} 1$. All the strains were characterized as $\mathrm{m} 1$ in the $v a c A$ mid-region using the primers VA3-F and VA3-R [8].
Sequence analysis: Sequence analysis of the $623 \mathrm{bp}$ fragment of the 5' end of the vacA gene showed the substitution of two bases in the nucleotide sequence corresponding to the 3' end of the complementary sequence of recognition for alignment of the forward primer (SS3-F). The substitutions prevented the binding of this primer and resulted in a lack of amplification of the PCR product, while the complementary nucleotide sequence for the binding site of the VA1-R reverse primer was completely conserved (Figure 2). All the sequences of the 5 selected strains showed the same nucleotide substitution at the 3'end of the complementary sequence of recognition for alignment of the forward primer (SS3-F).

The alignment of the remaining nucleotide sequences was more similar to $\mathrm{s} 1 \mathrm{~b}$ than $\mathrm{s} 1 \mathrm{a}$ or s1c. In order to find out if these nucleotide changes were present in all childhood strains (previously not characterized), one new primer SS5-F was designed and new PCRs were performed. We observed PCR products characterizing the signal sequence in all nonsubtypeable strains. To determine the specificity of this new primer, we performed individual PCR using SS5F and VA1-R primers with DNA of $60190,26690,84183$ and TX30a strains. This primer set did not amplify the vacA signal sequence of 60190 and 26690 (s1a), 84183 (s1b) and TX30a (s2) strains, indicating that the primer SS5-F is specific for the signal sequence of our strains, which we named as s1d subtype.

\section{A}

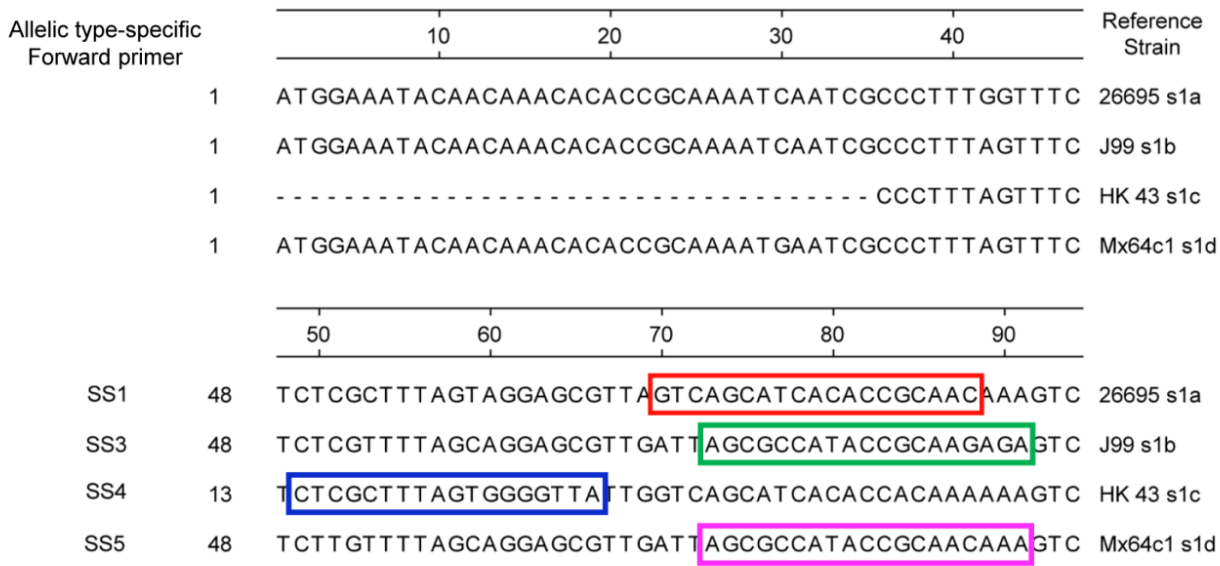

B

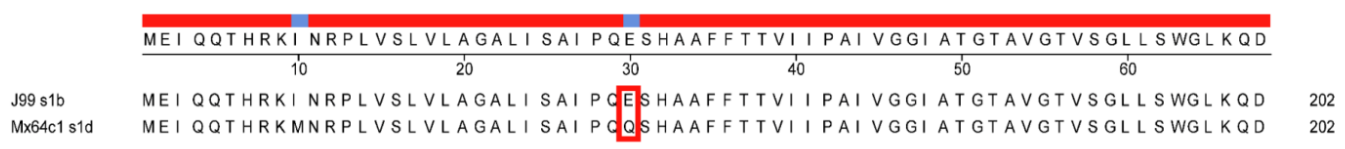

Figure 2: Alignment of nucleotide sequences (A) and amino acid sequences (B) of vacA signal sequence.

A. The red, green, blue and pink rectangles show the alignment site of each specific forward primer for each signal sequence subtype characterization. B. Amino acid substitutions between s1b and s1d, and the change of glutamic acid (E) by glutamine (Q) in position 30 is constant among all sequenced strains. 
In order to demonstrate that this was indeed a new subtype of signal sequences for the vacA gene, we performed phylogenetic analysis of 132 strains with different ss subtypes (s1a, s1b and s1c) including our own (s1d). The analyzed sequences were edited to ensure that all had the same size (132 nt). The editions were performed using the Seqman (DNASTAR Lasergene 7) and alignment was performed using the ClustalW2 program from Bioedit 7.0.9.0. The genealogy was reconstructed for the vacA ss of $H$. pylori using TCS software related to the Phylogenetic network estimation with statistical parsimony. Genealogic analysis of all the s1 signal sequence subtypes of 132 strains from different geographic origin was included (Figure 1). The haplotype network showed the formation of four main clades with a specific subtype grouping in each one, in which the s1d subtype formed an homogenous and compact cluster that diverged from the $\mathrm{s} 1 \mathrm{~b}$ signal sequence at two mutational steps and from $\mathrm{s} 2$ at 14 mutational steps (Figure 1a). This analysis revealed that there is recombination into the subpopulation between members of the same subtype but not between members of different subtypes. Considering the analysis of all the signal sequences available in the database, we can see that strains of s1d genotype derive from Colombia, Argentina, Peru and Venezuela, as well as one from Italy, indicating that this genotype has a distribution among Latin American populations predominantly (Figure 1b). This result supports previous reports in which the geographic distribution of H. pylori is linked to specific genotypes. This is the first study to report the presence of a new lineage (named here as sld subtype) in strains isolated from children with recurrent abdominal pain.

Structure analysis also supported the presence of three s1 subtypes (s1a, s1c, s1b/s1d), which were well defined and completely independent, each with specific polymorphic changes for each subpopulation (Figure 1c). However, the close genetic relationship between the $\mathrm{s} 1 \mathrm{~b}$ and s1d subtypes suggests that they share a common ancestor, with one giving rise to the other following very few mutational steps, as shown in Figure $1 \mathrm{~b}$.

With respect to the analysis of the middle region, the amplified alleles indicate a clear separation between $\mathrm{m} 1$ and $\mathrm{m} 2$ sequences (data not shown).

Vacuolating cytotoxin activity: From the $23 \mathrm{H}$. pylori isolates tested for vacuolating cytotoxin activity, broth culture supernatants of 13 strains caused vacuolation in AGS cells, while the remaining 10 strains did not produce vacuolating activity.

\section{Discussion}

H. pylori exhibits pronounced genetic diversity at both the chromosome and nucleotide sequence level in genes of the same locus from different strains [18-21]. This variability strongly impacts the virulence of the bacterium. Specific genotypes have been associated with the development of gastritis, peptic ulcers and stomach cancer [5-7,9-15,22]. The difference between genotypes of this bacterium is given by the presence or lack of gene blocks in the accessory genome within the plasticity zones of the chromosome and by the polymorphism of the nucleotide sequences in structural genes, such as the vacA gene, which encodes for the vacuolating cytotoxin [19-21]. H. pylori genotype
1 strains present the cag-PAI island and produce vacuolating cytotoxin with high activity. However, this association is only obvious in certain populations in which the infection is caused by only one type of strain, as seen in the United States and countries in Western Europe [5-7]. In contrast, there are other populations, in Japan and Korea for example, in which the characterization of strains isolated from both children and adults have shown the prevalence of $\operatorname{cag} A, \operatorname{vac} A \mathrm{~s} 1$, iceA1 genotype to be very high but with little or no relationship to gastro duodenal disease [22]. In Mexico, the relationship between the genotype of the strain and gastric pathology is not so clear. Several studies show that infection by this microorganism is really a multicolonization of the gastric mucosa by different $H$. pylori strains with different genotypes. Therefore, it is not possible to attribute a particular genotype to disease outcome [11-15]. These same studies also indicate the presence of strains that were not characterized according to their signal sequence, referring to these strains as being non-typeable. The relationship between recurrent abdominal pain and $H$. pylori infection is controversial. Some studies show that $H$. pylori is more frequent in patients with RAP compared to asymptomatic children, while others do not find an association between RAP and $H$. pylori infection [23-30]. H. pylori infection has also been reported with nonspecific symptoms, such as epigastric pain, dyspepsia, halitosis, nausea, vomiting and retrosternal pain. It is known that the prevalence of $H$. pylori infection among populations in different parts of the world varies with the highest levels being seen in developing countries where the infection is acquired during childhood. Epidemiological studies show that $H$. pylori infection is of mixed origin with strains of different genotypes of vacA, cag and iceA [11-15]. The coexistence of different strains in a bacterial population sharing only one niche leads to high rates of recombination, generating new genotypes among strains of the same species or different genders, which are better adapted to micro-environmental conditions $[2,18,31,32]$. The exchange of genetic material between bacteria is a common phenomenon in which the horizontal transference of genes participates through mobile elements, enriching the accessory genome that is suitable for the bacterium even more [33-37]. It is known that $H$. pylori is a natural transformant, which makes it genome dynamic. This process is necessary in response to stress conditions or selective pressures exerted by biochemical, physiological and anatomical factors in each region of the stomach [38,39].

In Mexico, infection by the bacterium is a chronic process with infection often being acquired during childhood. Colonization over an indefinite period of time is the result of well-developed strategies to avoid the immune response, competition for nutrients and to avoid over population in a specific niche $[31,40,41]$. We do not know why multiple infection in our patients is frequent. Two theories that could explain this phenomenon are: a) the strains that colonize the stomach present genetic exchange between them over years, thereby generating new strains, which are different from the original ones, or b) through the life (child-adult) of an individual, repeated infections with strains of different genotypes occur. Whatever the cause of mixed infection by $H$ pylori, certain questions arise, such as what is the role of 
each genotype in the development of disease when infection is caused by strains of different genotypes? Does synergism exist between different genotypic strains to produce more severe disease? Is there competition between different genotypic strains with the eventual abatement of a specific genotype? Does mixed infection lead to balance, controlling the development of disease? What is the result if this equilibrium is broken? Which genotype allows $H$. pylori to adapt in a better way? The predominance of which genotype allows it to be detected by gastric biopsy culture?

The phylogenetic and genealogic analysis of the nucleotide signal sequences of $v a c A$ showed that s1 and s2 genotypes diverge over 14 mutational steps (Figure 1a). Additionally, analysis of genotype s1 showed that each subtype (s1a, s1b, s1c and s1d) forms a coherent, robust and separate lineage, while s1d shows a close relationship to $\mathrm{s} 1 \mathrm{~b}$, suggesting a common ancestry for both these subtypes. The geographical distribution of the different $v a c A$ subtypes is well known with the $\mathrm{s} 1 \mathrm{~b}$ subtype being predominant in Hispanic countries. Meanwhile, s1d subtype distribution is almost exclusively seen in Latin America. Interestingly, the s1d Mexican haplotypes were isolated from children with recurrent abdominal pain. These results support the hypothesis suggested by Van Doorn, et al. that the distribution of the different vacA genotypes suggests a particular adaptation of the microorganism to specific host populations, as seen with the s1a genotype that is widely distributed in Northern and Eastern Europe, as well as in Canada and Australia, while the s1c subtype is more commonly found in East Asia.

According to the nucleotide sequence analysis, we can infer that the lineages of $s 2, s 1 b$ and sld were the ones who settled first in the stomach. It would be interesting to perform a genetic study and analysis in a single stomach following co-evolution of different genotypes over a long period of time in order to establish the original genotype and deduce its genetic changes until new subtypes are observed.

The results from this present study suggest that the first vacA genotypes of $H$. pylori established in the human stomach are not cytotoxic. Probably, the bacterium modified its genome generating cytotoxic genotypes (sla, slc), changing its status from non-pathogenic to pathogenic. This tends to support the hypothesis formulated by Blaser that "Helicobacter pylori is a commensal bacterium" and the acquisition of genetic material and mutations into its genome and individual genes make it a pathogenic bacterium [2, 31,40-42].

The present study does contain some limiting factors. The study group was small, limited to just a strains that could not be typed in a previous study. This made it impossible to determine any association between s1d type and RAP at a level with statistical significance. Although it is true that all the strains were isolated from children with RAP, it is necessary to study a larger number of strains isolated from children with RAP together with a larger control population (asymptomatic children) to find a real association with the $\mathrm{s} 1 \mathrm{~d}$ vacA signal sequence type.

In conclusion, our results show evidence of recombination between members of a population with the same subtype (Figure
1). However, recombination between members of different subtypes is rare, suggesting that each population of each subtype continues to colonize the stomach independently, which would explain the persistence of mixed infections. This phenomenon makes it difficult to associate one specific genotype to a given gastric pathology. A novel vacA s1d signal sequence type was found in Mexican children with recurrent abdominal pain. This type of signal sequence presented variable vacuolating activity. Further studies will be needed to determine if this vacA sequence signal subtype has important implications on the pathogenesis of digestive diseases. This sld subtype appears to be almost exclusively from Latin America. Finally, sequence analysis of each vacA signal sequence (s1a, s1b, s1c, and s1d) subtype demonstrated that each subtype forms a coherent, robust and separate subgroup.

\section{Conflict of interest}

This research was funded by DGAPA-PAPIIT Grant number IN213816 from Universidad Nacional Autónoma de México and CONACYT grant CB-255574. The funders had no role in study design, data collection and analysis, decision to publish, or preparation of the manuscript.

\section{References}

1. Blaser MJ, Atherton JC. Helicobacter pylori persistence: biology and disease. J Clin Invest. 2004;113(3):321-333.

2. Atherton JC, Blaser MJ. Coadaptation of Helicobacter pylori and humans: ancient history, modern implications. J Clin Invest. 2009;119(9):2475-2487.

3. Weeks DL, Eskandari S, Scott DR, Sachs G. A H+-gated urea channel: the link between Helicobacter pylori urease and gastric colonization. Science. 2000;287(5452):482-485.

4. Akopyants NS, Clifton SW, Kersulyte D, Crabtree JE, Youree BE, Reece CA, et al. Analyses of the cag pathogenicity island of Helicobacter pylori. Mol Microbiol. 1998;28(1):37-53.

5. Censini S, Lange C, Xiang Z, Crabtree JE, Ghiara P, Borodovsky M, et al. cag, a pathogenicity island of Helicobacter pylori, encodes type I-specific and disease-associated virulence. Genetics. 1996;93(25):14648-14653.

6. Evans DG, Queiroz DM, Mendes EN, Evans DJ. Helicobacter pylori cagA status and $\mathrm{s}$ and $\mathrm{m}$ alleles of vacA in isolates from individuals with a variety of $\mathrm{H}$. pylori-associated gastric diseases. J Clin Microbiol. 1998;36(11):3435-3437.

7. Blaser MJ, Perez-Perez GI, Kleanthous H, Cover TL, Peek RM, Chyou $\mathrm{PH}$, et al. Infection with Helicobacter pylori strains possessing cagA is associated with an increased risk of developing adenocarcinoma of the stomach. Cancer Res. 1995;55(10): 2111-2115.

8. Atherton JC, Cao P, Richard M. Peek J, Tummuru MKR, Blaser MJ, Cover TL. Mosaicism in Vacuolating cytotoxin Alleles of Helicobacter pylori. Association of specific vacA types with cytotoxin production and peptic ulceration. J Biol Chem. 1995;270(30):17771-17777.

9. Yamaoka Y, Kodama T, Gutierrez O, Kim JG, Kashima K, Graham DY. Relationship between Helicobacter pylori iceA, cagA, and vacA status and clinical outcome: studies in four different countries. J Clin Microbiol. 1999;37(7):2274-2279. 
10. Azuma T, Kato S, Zhou W, Yamazaki S, Yamakawa A, Ohtani M, et al. Diversity of $v a c A$ and cagA genes of Helicobacter pylori in Japanese children. Aliment Pharmacol Ther. 2004;20 Suppl 1:7-12.

11. Morales-Espinosa R, Castillo-Rojas G, Gonzalez-Valencia G, Ponce de León S, Cravioto A, Atherton JC, et al. Colonization of Mexican patients by multiple Helicobacter pylori strains with different vacA and cagA genotypes. J Clin Microbiol. 1999 ;37(9):3001-3004.

12. González-Valencia G, Atherton JC, Muñoz O, Dehesa M, la Garza AM Torres J. Helicobacter pylori vacA and cagA genotypes in Mexican Adults and Children. J Infect Dis. 2000;182(5):1450-1454

13. Morales Espinosa R, González Valencia G, Delgado G, Méndez JL, Torres J, Cravioto A. Frequency and characterization of vapD gene in Helicobacter pylori strains of different vacA and cag-PAI genotype. Bioquimia. 2008;33(2):43-50.

14.González-Vázquez R, Córdova-Espinoza MG, Escamilla-Gutiérrez A, Morales-Méndez I, Ochoa-Pérez SA, Armendáriz-Toledano F, et al. Frequency of virulence genes in mixed infections with Helicobacter pylori strains from a Mexican population. Rev Gastroenterol México. 2016;81(1):11-20.

15.López-Vidal Y, Ponce-de-León S, Castillo-Rojas G, Barreto-Zúñiga $\mathrm{R}$, Torre-Delgadillo A. High diversity of vacA and cagA Helicobacter pylori genotypes in patients with and without gastric cancer. PLoS One. 2008;3(12):e3849.

16.Sambrook J, Fritsch EF, Maniatis T. Molecular Cloning: a laboratory manual. New York. Cold Spring Harbor. 2001.

17. Cover TL, Dooley CP, Blaser MJ. Characterization of and human serologic response to proteins in Helicobacter pylori broth culture supernatants with vacuolizing cytotoxin activity. Infect Immun. 1990;58(3):603-610.

18. Aviles-Jimenez F, Letley DP, Gonzalez-Valencia G, Salama N, Torres J, Atherton JC. Evolution of the Helicobacter pylori Vacuolating Cytotoxin in a Human Stomach. J Bacteriol. J Bacteriol. 2004;186(15):5182 5185 .

19. Israel DA, Salama N, Krishna U, Rieger UM, Atherton JC, Falkow S, et al. Helicobacter pylori genetic diversity within the gastric niche of a single human host. Proc Natl Acad Sci U S A. 2001;98(25):1462514630.

20. Jiang Q, Hiratsuka K, Taylor DE. Variability of gene order in different Helicobacter pylori strains contributes to genome diversity. Mol Microbiol. 1996;20(4):833-842.

21. Suerbaum S, Achtman M. Evolution of Helicobacter pylori: the role of recombination. Trends Microbiol. 1999;7(5):182-184.

22. Ko JS, Kim KM, Oh YL, Seo JK. cagA, vacA, and iceA genotypes of Helicobacter pylori in Korean children. Pediatr Int. 2008;50(5):628 631

23. Yamaoka Y, Kodama T, Kita M, Imanishi J, Kashima K, Graham DY. Relationship of vacA Genotypes of Helicobacter pylori to cagA Status, Cytotoxin Production, and Clinical Outcome. Helicobacter. 1998;3(4):241-253.

24. Chong SKF, Lou Q, Zollinger TW, Rabinowitz S, Jibaly R, Tolia V, et al. The seroprevalence of Helicobacter pylori in a referral population of children in the United States. Am J Gastroenterol. 2003;98(10):2162 2168
25. Das BK, Kakkar S, Dixit VK, Kumar M, Nath G, Mishra OP. Helicobacter pylori infection and recurrent abdominal pain in children. J Trop Pediatr. 2003;49(4):250-252.

26. Ozen H, Dinler G, Akyon Y, Kocak N, Yuce A, Gurakan F. Helicobacter pylori Infection and Recurrent Abdominal Pain in Turkish Children. Helicobacter. 2001;6(3):234-238.

27.Zeyrek D, Zeyrek F, Cakmak A, Cekin A. Association of Helicobacter pylori and giardiasis in children with recurrent abdominal pain. Turkiye Parazitol Derg. 2008;32(1):4-7.

28. Spee LAA, Madderom MB, Pijpers M, van Leeuwen Y, Berger MY, Logan $\mathrm{R}$, et al. Association between Helicobacter pylori and gastrointestinal symptoms in children. Pediatrics. 2010;125(3):e651-669.

29. Selimoglu MA, Karabiber H, Otlu B, Yildirim O, Ozer A, Samdanci E. Correlation of clinical, endoscopic, and histological findings with virulence factors in children with Helicobacter pylori gastritis. Eur J Gastroenterol Hepatol. 2014;26(6):602-6.

30. Banatvala N, Mayo K, Megraud F, Jennings R, Deeks JJ, Feldman RA. The cohort effect and Helicobacter pylori. J Infect Dis. 1993;168(1):219221.

31. Blaser MJ. Helicobacters are indigenous to the human stomach: duodenal ulceration is due to changes in gastric microecology in the modern era. Gut. 1998;43(5):721-727.

32. Fraser C, Alm EJ, Polz MF, Spratt BG, Hanage WP. The bacterial species challenge: making sense of genetic and ecological diversity. Science. 2009;323(5915):741-746.

33. Lawrence JG. Gene transfer, speciation, and the evolution of bacterial genomes. Curr Opin Microbiol. 1999;2(5):519-23.

34. Darmon E, Leach DR. Bacterial genome instability. Microbiol Mol Biol Rev. 2014;78(1):1-39.

35. Kaper JB, Hacker J. Pathogenicity islands and other mobile virulence elements. Washington DC. American ASM Press. (Society of Microbiology). 1999.

36. Juhas M, Meer JR Van Der, Gaillard M, Harding RM, Hood DW, Crook DW. Genomic islands: tools of bacterial horizontal gene transfer and evolution. FEMS Microbiol Rev. 2009;33(2):376-393.

37. Hacker J, Kaper JB. Pathogenicity Islands and the Evolution of Microbes. Annu Rev Microbiol. 2000;54:641-679.

38. Hofreuter D, Odenbreit S, Haas R. Natural transformation competence in Helicobacter pylori is mediated by the basic components of a type IV secretion system. Mol Microbiol. 2001;41(2):379-391.

39. Israel DA, Lou AS, Blaser MJ. Characteristics of Helicobacter pylori natural transformation. FEMS Microbiol Lett. 2000 ;186(2):275-280.

40. Blaser MJ. Theodore E. Woodward Award: Global warming and the human stomach: microecology follows macroecology. Trans Am Clin Climatol Assoc. 2005;116:65-75.

41. Steinert M. Pathogen intelligence. Front Cell Infect Microbiol. 2014;4:8.

42. Lin D, Koskella B. Friend, foe: factors influencing the movement of the bacterium Helicobacter pylori along the parasitism-mutualism continuum. Evol Appl. 2015;8(1):9-22. 